Quim. Nova, Vol. 36, No. 4, 570-576, 2013

\title{
PROCESSAMENTO DE PLACAS DE CIRCUITO IMPRESSO DE EQUIPAMENTOS ELETROELETRÔNICOS DE PEQUENO PORTE
}

\author{
Sérgio de Souza Henrique Júnior, Felipe Pereira de Moura, Roger de Souza Correa e Júlio Carlos Afonso* \\ Departamento de Química Analítica, Instituto de Química, Universidade Federal do Rio de Janeiro, Av. Athos da Silveira Ramos, \\ 149, B1. A, 21941-909 Rio de Janeiro - RJ, Brasil
}

Cláudio Augusto Vianna e José Luiz Mantovano

Departamento de Química e Materiais Nucleares, Instituto de Engenharia Nuclear, Rua Hélio de Almeida, 75, 21941 -906 Rio de

Janeiro - RJ, Brasil

Recebido em 31/5/12; aceito em 5/10/12; publicado na web em 18/2/13

\begin{abstract}
PROCESSING OF PRINTED CIRCUIT BOARDS OF SMALL ELECTRICAL AND ELECTRONIC DEVICES. A hydrometallurgical process applicable to printed circuit boards of small electrical and electronic devices was developed. This involved three leaching steps $\left(60{ }^{\circ} \mathrm{C}, 2 \mathrm{~h}\right): 6 \mathrm{~mol} \mathrm{~L}^{-1} \mathrm{NaOH}, 6 \mathrm{~mol} \mathrm{~L}^{-1} \mathrm{HCl}$ and aqua regia. $\mathrm{NaOH}$ removed the resin and flame retardant that covered the circuit boards. $\mathrm{HCl}$ dissolved the most electropositive metals and a small amount of copper $(\sim 0.3 \mathrm{wt} \%)$. Aqua regia dissolved the noble metals. Silver precipitated as $\mathrm{AgCl}$. Gold and platinum were quantitatively extracted with pure methylisobutylketone and Alamine 336 (10\% vol. in kerosene), respectively. Slow evaporation of the raffinate crystallized $\mathrm{CuCl}_{2} \cdot 4 \mathrm{H}_{2} \mathrm{O}$ (89\% yield).
\end{abstract}

Keywords: waste electrical and electronic equipment (WEEE); waste management; metals recovery.

\section{INTRODUÇÃO}

O ritmo impressionante do crescimento da fabricação e consumo de equipamentos eletroeletrônicos (EEE), combinado à tendência à redução da vida útil das gerações dos diversos EEE (obsolescência programada $)^{1}$ leva à produção do chamado lixo eletroeletrônico ou resíduos de equipamentos elétricos e eletrônicos (REEE ou waste electrical and electronic equipment - WEEE), que assumiu proporções alarmantes em todo o mundo. Estimativas indicavam que, em 2010, haveria mais de 3 bilhões de computadores obsoletos em todo o mundo. ${ }^{2}$ Cerca de 1,5 bilhão de celulares são substituídos por ano. ${ }^{3}$ A Organização das Nações Unidas (ONU) estimava que a produção de lixo eletroeletrônico atingiria 150 milhões de t em $2010 .{ }^{4} \mathrm{O}$ lixo eletroeletrônico cresce a uma velocidade três a cinco vezes maior que a do lixo urbano. ${ }^{5}$

A produção cada vez maior e mais rápida de novas gerações de EEE traz dois grandes riscos: o elevado consumo dos recursos naturais empregados na fabricação destes e a destinação final inadequada. ${ }^{6}$ Por isso, o primeiro grande impacto do lixo eletroeletrônico não é o seu descarte, mas sim a extração dos insumos necessários à sua fabricação. Em 2008, foram geradas 149,2 mil t de lixo eletroeletrônico no Brasil. ${ }^{5}$ Estima-se que cerca de 96,8 mil t de computadores sejam descartados no país por ano, o que coloca o Brasil como um dos líderes entre os países emergentes. ${ }^{7}$ A Lei 12.305/2010 (Política Nacional de Resíduos Sólidos), prevê nos artigos 30 a 36 (Capítulo III, Seção II) a responsabilidade compartilhada de fabricantes, importadores, distribuidores e vendedores na logística reversa para os seguintes produtos pós-consumo: pilhas, baterias, pneus, óleos lubrificantes, lâmpadas, agrotóxicos e produtos eletroeletrônicos. ${ }^{8}$ Atualmente, há um grupo de trabalho (GT) no Conselho Nacional de Meio Ambiente (CONAMA) que busca estabelecer uma resolução para disciplinar a gestão dos REEE.

Os REEE são compostos por materiais os mais diversos, especialmente poliméricos e metálicos. Muitos desses materiais têm alto

*e-mail: julio@iq.ufrj.br valor agregado, ${ }^{9}$ sendo que a maioria destes se encontra nas placas de circuito impresso (PCI). ${ }^{10}$ As quantidades de metais valiosos são significativas considerando-se, por exemplo, que a concentração de ouro existente na PCI é superior à encontrada em fontes naturais desse elemento. . $^{3,11,12}$

O material que compõe a base, chamado laminado, de uma PCI pode ter diferentes composições, por exemplo, fenolite (papelão impregnado com resina fenólica), fibra de vidro, resinas epóxi, composite (mistura de resina fenólica com a fibra de vidro) e materiais cerâmicos. ${ }^{10,13} \mathrm{O}$ laminado é recoberto por uma fina camada de cobre. Os circuitos integrados e as outras partes eletrônicas são unidos ao laminado por soldagem por meio de uma liga eutética contendo chumbo e estanho. As conexões entre os componentes ocorrem do lado contendo cobre, através de caminhos condutores. ${ }^{10}$

A literatura sobre o processamento dos REEE aumentou extraordinariamente nos últimos 10 anos, com mais de 1000 trabalhos publicados, demonstrando a relevância deste tema na área de pesquisa e desenvolvimento. Os focos centrais desses trabalhos são impactos ambientais do descarte e da má gestão do lixo eletroeletrônico; impactos sobre a saúde das pessoas, particularmente as que manipulam esse lixo e os moradores vizinhos aos locais dessa manipulação e, processos de reciclagem. Recentemente foram publicados alguns artigos de revisão que abordam tanto a reciclagem quanto os impactos ambientais causados pelos REEE. ${ }^{12,14-17}$ Face à variabilidade do teor de metais nobres nos diferentes EEE, e da enorme variabilidade da composição das gerações dos mesmos, as estratégias para se recuperar esses elementos e outros componentes de elevado valor agregado dependem do tipo de EEE a ser processado, pois isso determinará o número de etapas necessárias para se atingir o objetivo desejado. ${ }^{18} \mathrm{~A}$ presença de chumbo na solda, da fibra de vidro e de aditivos antichama bromados faz com que os processos de recuperação de componentes das PCI mereçam atenção redobrada em relação aos riscos à saúde dos trabalhadores envolvidos. O elevado nível de poluição causado por esses tipos de resíduos em alguns países da África, Ásia e América do Sul tem despertado uma crescente preocupação de governos e órgãos ambientais. ${ }^{10}$ 
Os processos para reciclagem de uma PCI podem ser mecânicos ${ }^{19,20}$ (cominuição, classificação e separação), químicos ${ }^{21,22}$ (pirometalurgia, hidrometalurgia, eletrometalurgia e biometalurgia) ou térmicos. ${ }^{10} \mathrm{O}$ tratamento mecânico é o menos agressivo ao meio ambiente e à saúde ocupacional, por gerar menos resíduos contaminantes. ${ }^{10,11}$ Em geral, após a moagem prévia das PCI seguem-se separações gravimétricas (diferenças de densidade), ${ }^{23-25}$ eletrostáticas e magnéticas. ${ }^{26-30}$ A moagem tem como desvantagens a geração de finos, que podem implicar em perdas de componentes de alto valor agregado (como os metais nobres), além de exigir maiores cuidados com a higiene e saúde ocupacional no local de trabalho e o elevado consumo energético. ${ }^{10}$ As frações contendo metais são processadas por métodos piro e/ou hidrometalúrgicos. Um dos maiores problemas dos processos pirometalúrgicos (calcinações, pirólises ${ }^{10}$ ) é a possibilidade de emissão de compostos tóxicos como, por exemplo, as dioxinas liberadas pela queima dos polímeros clorados. ${ }^{31}$ Algumas das vantagens dos processos hidrometalúrgicos em relação à pirometalurgia, são a economia de energia e o menor impacto ambiental. ${ }^{28}$ As frações metálicas são tratadas com soluções oxidantes ácidas ou alcalinas, seguidas de etapas de separação como filtração, extração por solventes, destilação e precipitação dos metais dissolvidos. Uma das tentativas atuais é o desenvolvimento de reagentes que dissolvam seletivamente metais nobres, devido à formação de complexos bastante estáveis. Cianetos, haletos, tioureia e tiossulfato são dos ligantes mais testados para essa finalidade, ${ }^{1}$ mas ainda é necessária muita pesquisa para se chegar a um resultado prático. A gestão dos resíduos finais gerados nos estudos da literatura não é muito citada.

Este trabalho visou desenvolver uma rota hidrometalúrgica sequencial de processamento de PCI de EEE de pequeno porte, que são matrizes mais simples que as de PCI de computadores, focando a recuperação de elementos de elevado valor agregado (metais nobres) presentes nesses materiais. A base desse processo é a dissolução seletiva de metais mais eletropositivos que o hidrogênio na série de potenciais em meio ácido não oxidante, seguida da dissolução dos menos eletropositivos em meio ácido oxidante. Essa proposta aparentemente não encontra paralelo na literatura, a qual geralmente relata a lixívia em uma única etapa em meio ácido oxidante. Além disso, não foi empregado nenhum processo de moagem, procedimento típico descrito na literatura. Em vez disso, foi feita uma etapa prévia de remoção da resina e do retardante de chama para facilitar o acesso dos reagentes aos componentes das PCI. Após a caracterização química dos lixiviados, foi feito o processamento dos mesmos com base em técnicas clássicas de separação (precipitação seletiva, extração por solventes).

\section{PARTE EXPERIMENTAL}

Foram empregadas 8 PCI de telefones celulares, 4 de um mesmo modelo (fabricante A) e 4 de um outro modelo (fabricante B), todas fabricadas em 2008. As PCI não sofreram qualquer tipo de pré-tratamento.

\section{Processamento sequencial das placas de circuito impresso}

\section{Etapa 1: tratamento com $\mathrm{NaOH}$}

As placas foram colocadas em um béquer sobre placa aquecedora-agitadora, em capela, sendo em seguida cobertas com solução de $\mathrm{NaOH} 6 \mathrm{~mol} \mathrm{~L}^{-1}$ (10 mL g ${ }^{-1}$ placa). A mistura reacional foi aquecida a $60{ }^{\circ} \mathrm{C}$, sob agitação magnética $(100 \mathrm{rpm})$ durante $1-4 \mathrm{~h}$. Após o período estipulado, a placa tratada foi retirada com o auxílio de uma pinça e lavada com água. As águas de lavagem e a solução alcalina, de coloração marrom turva, foram reunidas e filtradas em papel de filtro (filtração média) para isolamento da porção insolúvel, que foi lavada com água $\left(4 \mathrm{~mL} \mathrm{~g}^{-1}\right)$. A massa insolúvel foi seca ao ar. Parte da solução alcalina foi neutralizada com $\mathrm{H}_{2} \mathrm{SO}_{4} 6 \mathrm{~mol} \mathrm{~L}^{-1}$ e lentamente evaporada sob placa aquecedora a $\sim 50{ }^{\circ} \mathrm{C}$ em capela até a secura, obtendo-se um sólido pardo.

\section{Etapa 2: tratamento com $\mathrm{HCl}$}

As placas tratadas com solução de $\mathrm{NaOH}$ foram imersas em béquer sobre placa aquecedora-agitadora, em capela, sendo em seguida cobertas com solução de $\mathrm{HCl} 6 \mathrm{~mol} \mathrm{~L}^{-1}\left(5 \mathrm{~mL} \mathrm{~g}^{-1}\right.$ placa). A temperatura foi fixada em $60^{\circ} \mathrm{C}$ (100 rpm) e o tempo de reação foi variado entre 1 e $4 \mathrm{~h}$. Findo esse período, a placa foi retirada com o auxílio de uma pinça e lavada com água $\left(3 \mathrm{~mL} \mathrm{~g}^{-1}\right)$. As águas de lavagem e a solução ácida, de coloração verde-marrom, foram reunidas e filtradas em papel de filtro (filtração média) para isolamento da porção insolúvel, de tonalidade marrom-amarelada, que foi lavada com água $\left(4 \mathrm{~mL} \mathrm{~g}^{-1}\right)$. A massa insolúvel foi seca em estufa a $110^{\circ} \mathrm{C}$ por $2 \mathrm{~h}$.

\section{Etapa 3: tratamento com água régia}

As placas oriundas da etapa anterior foram colocadas em recipiente de vidro sobre placa aquecedora-agitadora, em capela, sendo em seguida cobertas com água régia $\left(\mathrm{HCl}+\mathrm{HNO}_{3}\right.$ concentrados, $3: 1 \mathrm{v} / \mathrm{v}$, $5 \mathrm{~mL} \mathrm{~g}^{-1}$ placa). O recipiente foi coberto por uma tampa contendo duas aberturas: uma para a entrada de ar e outra para a saída do efluente gasoso. A temperatura foi fixada em $60{ }^{\circ} \mathrm{C}$ (100 rpm) e o tempo de reação foi variado entre 1 e $4 \mathrm{~h}$. Ar foi admitido durante o processo (10 $\mathrm{mL} \min ^{-1}$ ) para remover o $\mathrm{NO}_{x}$ gerado no processo. Findo o tempo de reação, a tampa foi removida, a placa foi retirada com o auxílio de uma pinça e lavada com água $\left(3 \mathrm{~mL} \mathrm{~g}^{-1}\right)$. As águas de lavagem e a solução ácida, de coloração verde-turquesa, foram reunidas e centrifugadas para isolamento da porção insolúvel, de coloração esbranquiçada, que foi lavada com água ( $\left.4 \mathrm{~mL} \mathrm{~g}^{-1}\right)$ e centrifugada uma segunda vez. A massa insolúvel foi seca ao ar e ao abrigo da luz.

\section{Isolamento dos metais nobres}

Dadas as características dos agentes lixiviantes empregados nas três etapas sequenciais, e de acordo com os estudos da literatura, ${ }^{12,14-16}$ prevê-se que os metais nobres presentes nas PCI se acham presentes na lixívia com água régia e em seu resíduo insolúvel.

\section{Isolamento do ouro}

Esse elemento foi extraído com metil-isobutilcetona pura (razão fase aquosa/fase orgânica $=1 / 1 \mathrm{v} / \mathrm{v}$ ) a $25^{\circ} \mathrm{C}$, e isolado como $\mathrm{AuCl}_{3}$. $\mathrm{H}_{2} \mathrm{O}$ segundo procedimento descrito na literatura. ${ }^{32}$

\section{Isolamento da platina}

O metal foi extraído com Alamina 336 (10\% vol. em querosene, razão fase aquosa/fase orgânica $=1 / 1 \mathrm{v} / \mathrm{v}$ ) a $25^{\circ} \mathrm{C}$, e isolado como platina metálica conforme procedimento descrito na literatura. ${ }^{33}$

\section{Isolamento da prata}

$\mathrm{O}$ resíduo insolúvel em água régia foi tratado com solução de $\mathrm{NH}_{4} \mathrm{OH} 2 \mathrm{~mol} \mathrm{~L}^{-1}$ a $25^{\circ} \mathrm{C}\left(2 \mathrm{~mL} \mathrm{~g}^{-1}\right)$. A solução, incolor, foi separada do sólido insolúvel por centrifugação. O sólido foi lavado com água $\left(2 \mathrm{~mL} \mathrm{~g}^{-1}\right)$ e novamente centrifugado. As águas de lavagem foram juntadas à solução amoniacal.

\section{Isolamento do cobre}

Após a extração do ouro e da platina, a solução ácida residual (rafinado) foi lentamente evaporada sob placa aquecedora, em capela, a $\sim 50^{\circ} \mathrm{C}$ até $4-10 \%$ do volume inicial, empregando o mesmo recipiente usado no tratamento das PCI com água régia. Após imersão em banho de gelo por 10 min, obteve-se um sólido verde e uma solução 
Tabela 1. Comportamento das PCI* frente ao tratamento com solução de $\mathrm{NaOH} 6 \mathrm{~mol} \mathrm{~L}^{-1}\left(60^{\circ} \mathrm{C}\right)$ em função do tempo

\begin{tabular}{|c|c|c|c|c|c|}
\hline & $0 \mathrm{~h}$ & $1 \mathrm{~h}$ & $2 \mathrm{~h}$ & $3 \mathrm{~h}$ & $4 \mathrm{~h}$ \\
\hline Massa da placa tratada $(\mathrm{g})$ & $\begin{array}{l}6,33 \pm 0,17 \\
9,13 \pm 0,27\end{array}$ & $\begin{array}{l}6,23 \pm 0,17 \\
8,88 \pm 0,17\end{array}$ & $\begin{array}{l}6,14 \pm 0,10 \\
8,81 \pm 0,10\end{array}$ & $\begin{array}{l}6,11 \pm 0,03 \\
8,82 \pm 0,07\end{array}$ & $\begin{array}{l}6,12 \pm 0,11 \\
8,83 \pm 0,21\end{array}$ \\
\hline Massa de resíduo insolúvel (g) & $\begin{array}{l}0 \\
0\end{array}$ & $\begin{array}{l}0,11 \pm 0,01 \\
0,25 \pm 0,02\end{array}$ & $\begin{array}{l}0,19 \pm 0,02 \\
0,32 \pm 0,02\end{array}$ & $\begin{array}{l}0,23 \pm 0,03 \\
0,30 \pm 0,02\end{array}$ & $\begin{array}{l}0,20 \pm 0,01 \\
0,30 \pm 0,03\end{array}$ \\
\hline
\end{tabular}

* Média de 4 experimentos para cada grupo de PCI de um mesmo fabricante.

de coloração verde intensa. O sólido foi filtrado em funil de Büchner sob vácuo e imediatamente colocado em frasco de vidro seco com rolha esmerilhada, sendo conservado em dessecador.

\section{Métodos analíticos}

Os elementos presentes em solução (aquosa e orgânica) e nos produtos finais sólidos obtidos foram analisados por fluorescência de raios-x por dispersão de comprimento de onda (WDXRF), técnica apropriada para a análise multielementar em matrizes complexas, como a deste estudo. Pipetaram-se $200 \mu \mathrm{L}$ de solução ou foram colocados $10 \mathrm{mg}$ de sólido em um papel de filtro de tamanho específico para o aparelho; o papel foi seco sob lâmpada incandescente ( $300 \mathrm{~W})$ e coberto com filme de poliéster. Inicialmente, efetuaram-se análises qualitativas ("varredura") com as amostras para identificação dos elementos presentes. Em seguida, foram estabelecidas curvas analíticas na faixa de 0,01 a $1,0 \mathrm{~g} \mathrm{~L}^{-1}$ dos elementos identificados em meio de $\mathrm{HCl} \mathrm{1,0} \mathrm{mol} \mathrm{L-1} \mathrm{(limite} \mathrm{de} \mathrm{quantificação:} 10 \mathrm{mg} \mathrm{kg}^{-1}$ ou $10 \mathrm{mg} \mathrm{L}^{-1}$ ). As placas antes e após cada etapa de processamento, bem como os produtos finais sólidos obtidos, foram pesados em balança analítica.

O sólido obtido após evaporação de parte da solução de $\mathrm{NaOH}$ (etapa 1) foi analisado por espectrosocpia de infravermelho (Nicolet 6700 FTIR) com amostra prensada em $\mathrm{KBr}$ a $2 \%$.

\section{RESULTADOS E DISCUSSÃO}

\section{Etapa de tratamento com solução de $\mathrm{NaOH}$}

Após a retirada das placas do béquer contendo a solução de $\mathrm{NaOH}$ 6 mol. $L^{-1}$, esta se apresentava bastante turva e tinha coloração marrom. Era possível ver pequenos pedaços provenientes da resina em suspensão. Poucos componentes soldados às placas se desprenderam das mesmas. Segundo os dados da Tabela 1, com base nas massas das placas antes e após o tratamento e nas massas de resíduos insolúveis obtidas, foram necessárias pelo menos $2 \mathrm{~h}$ para a remoção da resina que recobre as PCI. $2-3 \% \mathrm{~m} / \mathrm{m}$ da massa inicial da placa foi removida nesta etapa. A placa sem a resina tinha aspecto bem mais fosco e a lâmina de cobre aposta em um de seus lados tinha o brilho característico desse metal.

A análise da solução alcalina por WDXRF (Tabela 2) indicou que não havia quantidades significativas de metais dissolvidos, de modo que se pôde concluir que o tratamento com $\mathrm{NaOH}$ teve efeito basicamente apenas sobre a resina que recobre as placas, constituindo-se num pré-tratamento adequado de placas não trituradas para a rota hidrometalúrgica proposta neste trabalho. O sólido obtido após evaporação de parte da solução alcalina neutralizada com $\mathrm{H}_{2} \mathrm{SO}_{4}$ era totalmente solúvel em água e seu espectro de IV correspondia ao sal $\mathrm{Na}_{2} \mathrm{SO}_{4} \cdot 10 \mathrm{H}_{2} \mathrm{O}$. Quanto à massa insolúvel formada no curso do pré-tratamento, os dados da Tabela 2 indicam que apenas uma pequena porção da solda foi atacada e os componentes principais fazem parte da composição do laminado. ${ }^{13}$ Por outro lado, a presença de bromo indica a remoção do retardante de chama bromado da placa. A análise deste sólido por IV indica a presença de bandas características de amidas, anéis aromáticos, compostos carbonilados, ligação $\mathrm{C}-\mathrm{Br}$, ligação éter e carbonos alifáticos. Portanto, o resíduo insolúvel em $\mathrm{NaOH}$ consiste de uma fração orgânica e de uma fração inorgânica.

Tabela 2. Composição do resíduo insolúvel e da solução de $\mathrm{NaOH}^{*}$

\begin{tabular}{lll}
\hline Amostra & \multicolumn{2}{c}{$\begin{array}{c}\text { Composição }(\% \mathrm{~m} / \mathrm{m} \text { [sólidos]) ou } \\
\text { concentração }\left(\mathrm{mg} \mathrm{L}^{-1} \text { [soluções] }\right)\end{array}$} \\
\hline Resíduo insolúvel & $\mathrm{SiO}_{2}: 57,3 \pm 0,9$ & $\mathrm{CaO}: 0,6 \pm 0,2$ \\
& $\mathrm{BaO}_{2}: 25,0 \pm 1,5$ & $\mathrm{Br}: 0,4 \pm 0,1$ \\
& $\mathrm{SO}_{3}: 10,4 \pm 0,7$ & $\mathrm{SrO}: \sim 0,1$ \\
& $\mathrm{Al}_{2} \mathrm{O}_{3}: 2,7 \pm 0,5$ & $\mathrm{PbO}: \sim 0,1$ \\
& $\mathrm{Fe}_{2} \mathrm{O}_{3}: 1,8 \pm 0,3$ & $\mathrm{ZnO}, \mathrm{NiO}, \mathrm{SnO}_{2}:<0,1$ \\
& $\mathrm{MgO}: 0,6 \pm 0,1$ & \\
\hline Solução de $\mathrm{NaOH}$ & $\mathrm{Al}: 395 \pm 20$ & \\
& $\mathrm{Si}: 375 \pm 15$ & \\
& $\mathrm{Sn}: 10 \pm 2$ & \\
\hline
\end{tabular}

* Resultados referentes ao conjunto dos experimentos com as 8 PCI dos dois fabricantes.

\section{Lixiviação com $\mathrm{HCl}$}

O extrato clorídrico apresentava-se bastante turvo durante o procedimento, indicativo de sólidos em suspensão. 14,6 \pm 0,7\% $\mathrm{m} / \mathrm{m}$ da massa inicial das placas foram removidas nesta etapa. Os resíduos insolúveis eram de dois tipos: quase todos os componentes afixados às placas (capacitores, leds, indutores etc.) e, um sólido fino marrom claro. Após a separação dos componentes maiores, a análise do resíduo fino por WDXRF (Tabela 3) indicou a presença grandemente majoritária de componentes do laminado: silício, estrôncio, bário, cálcio, titânio, alumínio, ${ }^{13}$ um pouco de componentes da solda (chumbo e estanho) e, ainda, bromo, indicativo da presença de retardante de chama não removido pelo tratamento anterior com $\mathrm{NaOH}$. Na solução clorídrica (Tabela 3) os metais presentes em solução eram os mais eletropositivos do que o hidrogênio na série de potenciais, destacando-se os da solda ( $\mathrm{Pb}, \mathrm{Sn}$ - o que explica o desprendimento dos componentes afixados às placas) e ainda $\mathrm{Ni}, \mathrm{Co}, \mathrm{Al}, \mathrm{Ca}, \mathrm{Zn}$ e Fe (este último explica a coloração verde-amarela do lixiviado ${ }^{34}$ - íons $\mathrm{Fe}^{2+}$ e $\left.\mathrm{Fe}^{3+}\right)$. Pequenas quantidades de $\mathrm{Cu}(\sim 0,3 \%$ do cobre inicial) também estavam presentes nesta solução. Após $2 \mathrm{~h}$ de digestão das placas com $\mathrm{HCl}$ a concentração dos elementos não sofreu mudança significativa (Tabela 3), bem como a massa de insolúveis gerada nesse procedimento ( $\sim 30 \mathrm{mg} \mathrm{g}^{-1}$ placa). Os resultados são comparáveis para todas as placas examinadas.

Com base na caracterização química dos metais presentes nesse lixiviado (Tabela 3), apenas o cobre e o níquel apresentam concentrações que justificam uma tentativa de fracionamento. A solução ácida foi lentamente vertida em $\mathrm{NH}_{4} \mathrm{OH}$ concentrado $\left(15 \mathrm{~mol} \mathrm{~L}^{-1}\right.$, $\mathrm{pH}$ final $\sim 8$ ), a fim de manter o cobre e o níquel solúveis como aminocomplexos; ${ }^{34,35}$

$\mathrm{Ni}^{2+}+6 \mathrm{NH}_{4} \mathrm{OH} \rightleftarrows\left[\mathrm{Ni}\left(\mathrm{NH}_{3}\right)_{6}\right]^{2+}+6 \mathrm{H}_{2} \mathrm{O}$

$\mathrm{Cu}^{2+}+4 \mathrm{NH}_{4} \mathrm{OH} \rightleftarrows\left[\mathrm{Cu}\left(\mathrm{NH}_{3}\right)_{4}\right]^{2+}+4 \mathrm{H}_{2} \mathrm{O}$

os demais metais precipitaram como hidróxidos, salvo o zinco. Mais de $99,5 \%$ dos três elementos permaneceram na solução amoniacal. 
Tabela 3. Composição do resíduo insolúvel e concentrações no lixiviado com $\mathrm{HCl}\left(60^{\circ} \mathrm{C}, 2 \mathrm{~h}\right)^{*}$ após tratamento prévio das PCI com solução de $\mathrm{NaOH}$ $6 \mathrm{~mol} \mathrm{~L}^{-1}\left(60^{\circ} \mathrm{C}, 2 \mathrm{~h}\right)$

\begin{tabular}{ccc}
\hline Elemento & Resíduo insolúvel $(\% \mathrm{~m} / \mathrm{m})$ & Solução $\left(\mathrm{mg} \mathrm{L}^{-1}\right)^{* * * *}$ \\
\hline $\mathrm{Si}$ & $87,6 \pm 1,9$ & $\mathrm{nd}^{* * *}$ \\
$\mathrm{Al}$ & $6,0 \pm 0,9$ & $<10^{* * *}$ \\
$\mathrm{~S}$ & $2,6 \pm 0,1$ & $200 \pm 10$ \\
$\mathrm{Ca}$ & $1,8 \pm 0,3$ & $330 \pm 30$ \\
$\mathrm{Fe}$ & $1,3 \pm 0,2$ & $4560 \pm 120$ \\
$\mathrm{Ba}$ & $0,3 \pm 0,1$ & $<10^{* *}$ \\
$\mathrm{Ti}$ & $\sim 0,1$ & $\mathrm{nd}$ \\
$\mathrm{Cu}$ & $<0,1$ & $60 \pm 10$ \\
$\mathrm{Br}$ & $\sim 0,1$ & $\mathrm{nd}$ \\
$\mathrm{Pb}$ & $\sim 0,1$ & $1260 \pm 20$ \\
$\mathrm{Sn}$ & $\sim 0,1$ & $3090 \pm 20$ \\
$\mathrm{Ni}$ & nd & $1650 \pm 30$ \\
$\mathrm{Zn}$ & nd & $2200 \pm 40$ \\
\hline
\end{tabular}

* Massa média da placa removida pelo tratamento com $\mathrm{HCl}$ : 14,6 $\pm 0,7 \%$ $\mathrm{m} / \mathrm{m}(0,924 \mathrm{~g}$ para as placas do fabricante A e $1,47 \mathrm{~g}$ para as do fabricante B). **Não detectado (limite de detecção $1 \mathrm{mg} \mathrm{kg}^{-1}$ ou $1 \mathrm{mg} \mathrm{L}^{-1}$ ). Os dados para o resíduo insolúvel são expressos na forma de óxido. ***Detectado, mas não quantificado (limite de quantificação $10 \mathrm{mg} \mathrm{L}^{-1}$ ). ****Volume médio (lixiviante + águas de lavagem): $80 \mathrm{~mL}$.

Após acidificação com $\mathrm{HCl} 6 \mathrm{~mol} \mathrm{~L}^{-1}$, o cobre foi isolado (> 99,9\%) mediante adição de $\mathrm{Na}_{2} \mathrm{~S}$ e o níquel precipitado como $\mathrm{Ni}(\mathrm{OH})_{2}(>$ $99,5 \%$ ) mediante adição de solução de $\mathrm{NaOH} 6 \mathrm{~mol} \mathrm{~L}^{-1}$ (pH 14). ${ }^{36}$ $\mathrm{O}$ zinco permaneceu solúvel como $\left[\mathrm{Zn}(\mathrm{OH})_{4}\right]^{2-}$. Após neutralização com $\mathrm{HCl} 6 \mathrm{~mol} \mathrm{~L}^{-1}$, o hidróxido $\mathrm{Zn}(\mathrm{OH})_{2}$ precipitou $^{34,35}(>99,5 \%) \mathrm{e}$ a solução final continha uma mistura de $\mathrm{NH}_{4} \mathrm{Cl}$ e $\mathrm{NaCl}$.

Embora o processo de fracionamento de níquel, cobre e zinco seja factível como descrito acima, exige o emprego de reagentes tóxicos $\left(\mathrm{Na}_{2} \mathrm{~S}\right)$ e gera um efluente final salino contendo $\mathrm{NH}_{4}^{+}$, poluente ambiental. ${ }^{37}$ Uma rota alternativa de fracionamento é a extração desses metais por meio de extratantes dissolvidos em solventes orgânicos. ${ }^{1,22}$

\section{Comportamento da lixívia com água régia}

A água régia dissolve o ouro, a platina e o cobre e reage com a prata segundo as reações ${ }^{34,35}$

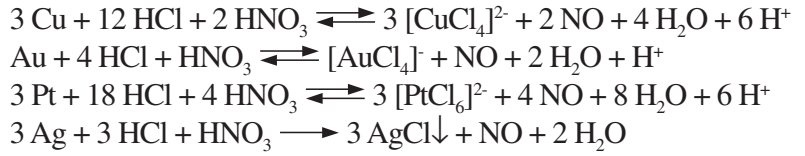

$\mathrm{O} \mathrm{NO}$ é rapidamente convertido em $\mathrm{NO}_{2}$ (gás de coloração castanha) pelo oxigênio atmosférico. Após a evolução de todo o $\mathrm{NO}_{2}$ produzido, a solução adquiriu tonalidade verde-turquesa relativa ao clorocomplexo de cobre (os de platina e de ouro têm coloração amarela brilhante). A prata precipita como um sólido branco insolúvel no meio reacional. $28,8 \pm 0,8 \% \mathrm{~m} / \mathrm{m}$ da placa inicial foram removidas por meio deste tratamento oxidativo após pelo menos $2 \mathrm{~h} \mathrm{a} 60^{\circ} \mathrm{C}$.

A Tabela 4 mostra os dados de composição do lixiviado e do precipitado obtidos após o tratamento com água régia. Do mesmo modo que no caso com $\mathrm{HCl}$, os resultados variaram muito pouco entre as placas analisadas. Esse lixiviado tinha largo predomínio de cobre (mais de $99 \%$ do total dos metais e $99,7 \% \mathrm{~m} / \mathrm{m}$ do elemento inicialmente presente nas placas). Além de ouro, aparecem também pequenas quantidades de $\mathrm{Fe}, \mathrm{Ni}, \mathrm{Al}, \mathrm{Sn}, \mathrm{Ca}$ e $\mathrm{Co}$. A platina apareceu em 4 das PCI. Quanto ao sólido, ele era basicamente composto apenas por cloreto de prata e sílica, desprendida da placa. A prata pode ser separada por adição de hidróxido de amônio $\left(\mathrm{NH}_{4} \mathrm{OH}\right)^{: 34,35}$

$\mathrm{AgCl}+2 \mathrm{NH}_{4} \mathrm{OH} \rightleftarrows \mathrm{Ag}\left(\mathrm{NH}_{3}\right)_{2} \mathrm{Cl}+2 \mathrm{H}_{2} \mathrm{O}$

Com base nos dados da Tabela 4 e na diferença de massa do resíduo sólido antes e depois do tratamento com $\mathrm{NH}_{4} \mathrm{OH}$, o $\mathrm{AgCl}$ compunha cerca de $60 \%$ da massa do mesmo.

Tabela 4. Composição do resíduo insolúvel e concentrações no lixiviado com água régia $\left(60^{\circ} \mathrm{C}, 2 \mathrm{~h}\right) *$ após tratamento prévio das $\mathrm{PCI}$ com solução de $\mathrm{NaOH} 6 \mathrm{~mol} \mathrm{~L}^{-1}\left(60^{\circ} \mathrm{C}, 2 \mathrm{~h}\right)$ e com $\mathrm{HCl} 6 \mathrm{~mol} \mathrm{~L}^{-1}\left(60^{\circ} \mathrm{C}, 2 \mathrm{~h}\right)$

\begin{tabular}{ccc}
\hline Elemento & Resíduo insolúvel $(\% \mathrm{~m} / \mathrm{m})$ & Solução $\left(\mathrm{mg} \mathrm{L}^{-1}\right)^{* * * *}$ \\
\hline $\mathrm{Si}$ & $40 \pm 4$ & $\mathrm{nd}^{* *}$ \\
$\mathrm{Ag}$ & $60 \pm 4$ & $\mathrm{nd}$ \\
$\mathrm{Cu}$ & $\mathrm{nd}$ & $31900 \pm 250$ \\
$\mathrm{Ca}$ & $\mathrm{nd}$ & $110 \pm 30$ \\
$\mathrm{Fe}$ & $\mathrm{nd}$ & $520 \pm 50$ \\
$\mathrm{Ni}$ & $\mathrm{nd}$ & $30 \pm 10$ \\
$\mathrm{Sn}$ & $\mathrm{nd}$ & $15 \pm 5$ \\
$\mathrm{Au}$ & $\mathrm{nd}$ & $40 \pm 3$ \\
$\mathrm{Pt}$ & $\mathrm{nd}$ & $12 \pm 2 * * *$ \\
\hline
\end{tabular}

* Massa média da placa removida pelo tratamento com água régia: $28,8 \pm 0,8 \%$ $\mathrm{m} / \mathrm{m}(1,82 \mathrm{~g}$ para as placas do fabricante A e 2,63 g para as do fabricante B). **Não detectado (limite de detecção $1 \mathrm{mg} \mathrm{kg}^{-1}$ ou $1 \mathrm{mg} \mathrm{L}^{-1}$ ). Os dados para o resíduo insolúvel são expressos na forma de óxido. ***Detectado em apenas 4 das 8 PCI. ****Volume médio (lixiviante + águas de lavagem): $80 \mathrm{~mL}$.

\section{Recuperação de ouro, platina e cobre}

Em um único estágio (FA/FO = $1 \mathrm{vol} / \mathrm{vol})$ a $25^{\circ} \mathrm{C} 99,7 \pm 0,2 \%$ de ouro e 99,4 $\pm 0,1 \%$ da platina foram extraídos, respectivamente, com metilisobutilcetona e Alamina 336. Esse fato é explicável pela baixa concentração desses elementos nas soluções (Tabela 4). As fases orgânicas tinham coloração amarela intensa, enquanto que a fase aquosa final adquiria uma cor azul, referente aos íons $\mathrm{Cu}^{2+}$. Apenas traços de ferro foram identificados na solução orgânica contendo ouro. A elevada seletividade na extração do ouro e da platina se explica pela remoção prévia de elementos interferentes ( $\mathrm{Sn}, \mathrm{Pb}$ e $\mathrm{Fe}$ ) na etapa com $\mathrm{HCl}$. O desempenho das extrações de ouro e platina foi o mesmo verificado no caso dos cartuchos de impressoras de jato de tinta ${ }^{32}$ e catalisadores. ${ }^{33}$ Os seus produtos finais, $\mathrm{AuCl}_{3} \cdot \mathrm{H}_{2} \mathrm{O}^{32}$ e platina metálica, ${ }^{33}$ continham mais de $99,5 \%$ dos elementos originalmente presentes nas placas.

Após a extração do ouro e da platina, a evaporação da fase aquosa ácida para obtenção do $\mathrm{CuCl}_{2} \cdot 4 \mathrm{H}_{2} \mathrm{O}$ se mostrou um procedimento delicado, devido à natureza deliquescente desse sal. A primeira preocupação era determinar o limite da evaporação a fim de se obter o sólido desejado sem a intromissão dos outros metais presentes. Os dados da Tabela 5 indicam que o limite de evaporação foi de $95 \%$ do volume inicial, situação em que cerca de $89 \% \mathrm{~m} / \mathrm{m}$ do cloreto de cobre(II) cristalizou. A solução aquosa residual tinha coloração azul $\left(\mathrm{Cu}^{2+}\right)$ e continha como contaminantes $\mathrm{Fe}, \mathrm{Ni}, \mathrm{Ca}$ e traços de $\mathrm{Cr}$.

\section{Balanço da distribuição dos elementos identificados nas PCI}

A Tabela 6 mostra como se distribuíram os diversos elementos presentes nas PCI processadas. A solução de $\mathrm{NaOH}$ praticamente 
Tabela 5. Concentrações típicas na solução aquosa residual após a cristalização do sal $\mathrm{CuCl}_{2} \cdot 4 \mathrm{H}_{2} \mathrm{O}$ por evaporação de 92,5 ou $95 \%$ do volume inicial, e composição típica do $\mathrm{CuCl}_{2} \cdot 4 \mathrm{H}_{2} \mathrm{O} *$

\begin{tabular}{ccccc}
\hline \multirow{2}{*}{ Elemento } & \multicolumn{2}{c}{$\mathrm{CuCl}_{2} \cdot 4 \mathrm{H}_{2} \mathrm{O}(\% \mathrm{~m} / \mathrm{m})$} & \multicolumn{2}{c}{ Solução residual $\left(\mathrm{mg} \mathrm{L}^{-1}\right)$} \\
\cline { 2 - 5 } & $6 \mathrm{~mL}$ & $4 \mathrm{~mL}$ & $6 \mathrm{~mL}$ & $4 \mathrm{~mL}$ \\
\hline $\mathrm{Cu}^{* *}$ & 99,8 & 99,8 & 127500 & 67200 \\
$\mathrm{Ca}$ & $\mathrm{nd}$ & $\mathrm{nd}^{* * *}$ & 1480 & 2200 \\
$\mathrm{Fe}$ & 0,1 & 0,1 & 6850 & 10400 \\
$\mathrm{Ni}$ & $\mathrm{nd}$ & $\mathrm{nd}$ & 400 & 630 \\
$\mathrm{Sn}$ & 0,1 & 0,1 & $\mathrm{nd}$ & $\mathrm{nd}$ \\
\hline
\end{tabular}

*Valores médios obtidos para as 8 placas processadas (variação não superior a 5\%). **Rendimento em massa: $70 \%$ (evaporação a $6 \mathrm{~mL}$ ), $89 \%$ (evaporação a $4 \mathrm{~mL}$ ). Abaixo de $92,5 \%$ o sal não cristaliza e acima de $95 \%$ toda a massa residual de água é incorporada ao sólido. ***Não detectado (limite de detecção $1 \mathrm{mg} \mathrm{kg}^{-1}$ ou $1 \mathrm{mg} \mathrm{L}^{-1}$ ).

não continha os elementos assinalados $(<0,3 \%)$; a única ressalva é que ela continha praticamente todo o bromo presente na placa inicial. A lixívia com $\mathrm{HCl}$ concentrou a totalidade do chumbo, zinco e quase a totalidade do estanho (> 99\%), do níquel (> 98\%) e do ferro ( 90\%). Já a lixívia oxidante (água régia) continha a totalidade dos metais nobres (ouro, platina, prata) e quase todo o cobre (>99,5\%). Quanto aos elementos presentes no laminado, bário e estrôncio somente apareceram no lixiviado com $\mathrm{HCl}$, enquanto que cálcio, silício e alumínio também se acharam largamente presentes nesse lixiviado.

Tabela 6. Distribuição dos elementos nas lixívias com $\mathrm{HCl}$ e com água régia, e teores médios $(\% \mathrm{~m} / \mathrm{m})$ estimados para diversos elementos nas PCI

\begin{tabular}{cccc}
\hline Elemento & Lixívia com $\mathrm{HCl}$ & $\begin{array}{c}\text { Lixívia com } \\
\text { água régia }\end{array}$ & Teor médio* \\
\hline $\mathrm{Pb}$ & 100 & 0 & 1,11 \\
$\mathrm{Zn}$ & 100 & 0 & 1,93 \\
$\mathrm{Sn}$ & 99,2 & 0,8 & 2,73 \\
$\mathrm{Fe}$ & 89,8 & 10,2 & 4,50 \\
$\mathrm{Ni}$ & 98,2 & 1,2 & 1,47 \\
$\mathrm{Cu}$ & 0,3 & 99,7 & 27,95 \\
$\mathrm{Au}$ & 0 & 100 & $3,5 \times 10^{-2}$ \\
$\mathrm{Ag}$ & 0 & 100 & 0,21 \\
$\mathrm{Pt}$ & 0 & 100 & $9,0 \times 10^{-3}$ \\
$\mathrm{Ca}$ & 75,2 & 24,8 & nd \\
$\mathrm{Sr}$ & 100 & 0 & nd \\
$\mathrm{Ba}$ & 100 & 0 & nd \\
$\mathrm{Si}$ & 85,7 & 14,2 & nd \\
$\mathrm{Al}$ & 86,4 & 13,6 & nd \\
\hline
\end{tabular}

* Obtidos com base nos dados de composição apresentados nas Tabelas 3 a 5.

Outro dado de relevância foi a determinação do teor de elementos nas PCI processadas. Com base nos dados das Tabelas $3 \mathrm{e}$ 4, chega-se aos resultados apresentados na Tabela 6, Ao comparar esses resultados com os de uma referência recentemente publicada, ${ }^{12}$ a maioria dos teores se enquadra na faixa indicada na referência. A mudança constante da concepção das gerações de EEE faz com que os teores de certos elementos aumentem ou diminuam em relação às gerações anteriores, bem como novos elementos podem aparecer em substituição a outros..$^{2,412}$

\section{Gestão dos resíduos finais do processamento químico das placas}

Um dos aspectos mais relevantes quanto ao processamento do lixo eletroeletrônico é a sua natureza multicomponente. Mesmo quando se empregam métodos físicos tanto quanto possível, não há como evitar o emprego de etapas químicas para separação e isolamento de elementos de interesse e/ou de alto valor agregado. Isso se reflete diretamente na geração de resíduos de processo.

\section{Soluções aquosas}

A solução alcalina obtida na primeira etapa do processamento das PCI pode neutralizar a lixívia clorídrica, precipitando os metais em conjunto; o precipitado pode ser encaminhado para aterro industrial para resíduos classe I (norma brasileira NBR ABNT 10.004). ${ }^{38}$ A solução final contém $\mathrm{NaCl}$ e pode ser descartada segundo a Resolução 430/2011 do Conselho Nacional de Meio Ambiente (CONAMA). ${ }^{39}$ A solução ácida após o isolamento do composto $\mathrm{CuCl}_{2} \cdot 4 \mathrm{H}_{2} \mathrm{O}$ também pode ser neutralizada com a solução de $\mathrm{NaOH}$ usada no pré-tratamento das placas ${ }^{38} \mathrm{ou}$, ainda, ser reaproveitada para compor a solução ácida oxidante para tratamento de novas placas. A dificuldade nesse aspecto é a tendência ao aumento da concentração de outros elementos (Tabela 5), reduzindo o rendimento do isolamento do $\mathrm{CuCl}_{2} \cdot 4 \mathrm{H}_{2} \mathrm{O}$. Uma solução para contornar esse problema é extrair os elementos interferentes $(\mathrm{Fe}$, Sn) com extratantes dissolvidos em solventes orgânicos..$^{1,22}$

Sólidos

Existem diversos sólidos produzidos em todas as etapas do processamento das PCI. Com base nas características desses sólidos, eles podem ser combinados e enviados a coprocessamento, de conformidade com a Resolução 264/99 do CONAMA, ${ }^{40}$ atentando-se para os valores máximos permitidos para chumbo e bromo no blend preparado para essa destinação. Outro sólido relevante produzido é a PCI exaurida após os tratamentos sequenciais alcalino e ácidos. As análises de WDXRF não indicaram a presença de metais em quantidade acima do limite de detecção do método nesse resíduo sólido. Com base nesse dado, as PCI exauridas, que são o resíduo mais importante gerado no processo (cerca de $35 \%$ da massa inicial), também podem ser coprocessadas. ${ }^{40}$

\section{Efluentes gasosos}

Nesta categoria se enquadram o $\mathrm{NO}_{x}$ produzido na etapa de tratamento com água régia e o efluente produzido na evaporação para isolamento do $\mathrm{CuCl}_{2} \cdot 4 \mathrm{H}_{2} \mathrm{O}$. Com base na reação 3 , no teor de $\mathrm{Cu}$ nas PCI (Tabela 6) e na relação empregada $5 \mathrm{~mL}$ água régia $\mathrm{g}^{-1}$ PCI, estima-se que foram produzidos $0,14 \mathrm{~g}$ de $\mathrm{NO}_{2}$ por $\mathrm{g}$ de placa processada (140 $\left.\mathrm{g} \mathrm{kg}^{-1}\right)$, valor considerável, podendo ser classificado como o principal impacto ambiental do processo em discussão. Após a purga do meio reacional, o efluente gasoso contendo $\mathrm{NO}_{2}$ foi passado por solução de $\mathrm{NaOH} 6 \mathrm{~mol} \mathrm{~L}^{-1}$ à temperatura ambiente, onde ocorrem as reações:

$$
\begin{aligned}
& 2 \mathrm{NO}_{2}+2 \mathrm{NaOH} \longrightarrow \mathrm{NaNO}_{3}+\mathrm{NaNO}_{2}+\mathrm{H}_{2} \mathrm{O} \\
& \mathrm{HCl}+\mathrm{NaOH} \longrightarrow \mathrm{NaCl}+\mathrm{H}_{2} \mathrm{O}(9)
\end{aligned}
$$

Após a exaustão da solução alcalina, o nitrito pode ser oxidado a nitrato por adição de $\mathrm{H}_{2} \mathrm{O}_{2},{ }^{34,35}$ permitindo o descarte da mesma. ${ }^{39}$ $\mathrm{O}$ efluente da evaporação da solução ácida para isolamento do $\mathrm{CuCl}_{2} \cdot 4 \mathrm{H}_{2} \mathrm{O}$ era ácido devido à presença de $\mathrm{HCl}$, sendo também neutralizado pela solução de $\mathrm{NaOH}$ antes do descarte.

\section{Considerações sobre o custo do processamento das PCI}

Os custos do processo em exame se referem aos seguintes itens: 
- energia elétrica: aquecimento nas três etapas hidrometalúrgicas; cristalização do $\mathrm{CuCl}_{2} \cdot 4 \mathrm{H}_{2} \mathrm{O}$, isolamento do $\mathrm{AuCl}_{3} \cdot \mathrm{H}_{2} \mathrm{O}^{32}$ e da platina; ${ }^{33}$ filtração a vácuo do $\mathrm{CuCl}_{2} \cdot 4 \mathrm{H}_{2} \mathrm{O}$; centrifugação para separação da solução amoniacal de prata do $\mathrm{SiO}_{2}$;

- água: preparo das soluções de $\mathrm{NaOH} 6 \mathrm{~mol} \mathrm{~L}^{-1}$ (primeira etapa do processamento das $\mathrm{PCI}$, abatimento do $\mathrm{NO}_{2}$ separação $\mathrm{Ni} / \mathrm{Zn}$ ); preparo da solução de $\mathrm{HCl} 6 \mathrm{~mol} \mathrm{~L}^{-1}$ (segunda etapa do processamento, separação $\mathrm{Cu} / \mathrm{Ni} / \mathrm{Zn}$ ); lavagem dos resíduos insolúveis e dos precipitados obtidos nas três etapas de processamento (incluindo ouro ${ }^{32}$ e platina ${ }^{33}$ ) e da PCI exaurida;

- reagentes e solventes: $\mathrm{NaOH}, \mathrm{HCl}, \mathrm{HNO}_{3}$ (preparo da água régia), $\mathrm{NH}_{4} \mathrm{OH}$ (separação $\mathrm{AgCl}$ de $\mathrm{SiO}_{2}$, separação de $\mathrm{Ni} / \mathrm{Cu} /$ $\mathrm{Zn}$ da lixívia clorídrica, isolamento da platina $\left.{ }^{33}\right), \mathrm{Na}_{2} \mathrm{~S}_{2} \mathrm{O}_{3}$ e ácido ascórbico (isolamento da platina ${ }^{33}$ ), metil-isobutilcetona (isolamento do ouro ${ }^{32}$ ), Alamina 336 (isolamento da platina ${ }^{33}$ ), querosene (diluente para a Alamina $336^{33}$ ), $\mathrm{H}_{2} \mathrm{O}_{2}$ (oxidação de nitrito a nitrato $\left.{ }^{34,35}\right)$;

- destinação final de sólidos e de soluções aquosas.

Os produtos finais obtidos de alto valor agregado contêm metais nobres $(\mathrm{Au}, \mathrm{Ag}, \mathrm{Cu})$; salvo cobre, os demais são obtidos em pequenas quantidades após se processar uma PCI. Não se fez um estudo pormenorizado quanto a custos; apesar disso, nota-se que o grande número de etapas de fracionamento dos elementos nas PCI e de purificação/isolamento dos produtos finais dificulta sobremodo a viabilidade econômica deste e de quaisquer rotas similares ${ }^{1,19,28}$ para processar lixo eletroeletrônico. Esse fato provavelmente justifica o porquê da pesquisa de métodos de solubilização seletiva de metais preciosos. ${ }^{1,10,12}$ A extração, em meio ácido, de metais por meio de extratantes dissolvidos em solventes orgânicos (caso aplicado ao ouro e à platina neste trabalho) vem sendo apontada como uma alternativa para a redução da geração de resíduos, consumo de reagentes e de insumos. ${ }^{1,12,22}$

\section{CONCLUSÕES}

O pré-tratamento das PCI com solução de hidróxido de sódio se mostrou eficaz para remover a película que recobre as mesmas, praticamente sem a dissolução de metais, facilitando o ataque ácido nas etapas posteriores. Esse pré-tratamento cumpre o mesmo papel da moagem e trituração das PCI, que é o procedimento adotado na literatura.

A lixiviação ácida das placas em duas etapas permitiu obter uma solução contendo metais nobres contendo poucos elementos interferentes. A lixiviação inicial ( $1^{\mathrm{a}}$ etapa) com um ácido não oxidante $(\mathrm{HCl})$ dissolveu quase todos os metais mais eletropositivos do que o hidrogênio presentes nas amostras tratadas, e o sólido desprendido nessa etapa consiste basicamente de fibras de vidro. A $2^{\text {a }}$ etapa, conduzida em meio oxidativo (água régia), produziu uma solução muito rica em cobre e um precipitado contendo prata $(\mathrm{AgCl})$. Ouro e platina foram isolados dessa solução por meio de extrações líquido-líquido em série sem a interferência de outros elementos, com elevado rendimento e grau de pureza muito bom.

O processamento da lixívia clorídrica apresenta pouco interesse para o isolamento de metais dissolvidos, embora seja possível separar $\mathrm{Ni}, \mathrm{Cu}$ e Zn dos demais elementos. Outros métodos de separação vêm sendo testados para valorizar essa lixívia como matéria-prima para isolamento dos elementos presentes.

As vantagens da rota hidrometalúrgica em multietapas aplicadas a PCI de pequeno tamanho são evitar a moagem prévia das mesmas e a separação praticamente total dos elementos de maior valor agregado dos demais componentes das placas, facilitando o isolamento dos primeiros. Contudo, este processo não tem como ser aplicado a PCI de maior tamanho (como placas-mãe de computadores) devido à grande complexidade e heterogeneidade das mesmas.
Outra dificuldade é o elevado custo de um processo em multietapas (reagentes, solventes, insumos, tratamento de resíduos), dificultando sua viabilidade econômica.

Na qualidade de um material multicomponente, a PCI é um exemplo da necessidade de se conceber uma geração de um produto mais facilmente reciclável após o fim de sua vida útil. ${ }^{41}$

\section{MATERIAL SUPLEMENTAR}

Disponível gratuitamente na forma de arquivo pdf em http:// www.quimicanova/sbq.org.br, apresenta o fluxograma completo do processamento das placas de PCI.

\section{AGRADECIMENTOS}

Ao CNPq pelo auxílio financeiro. F. P. Moura e R. S. Correa agradecem ao Programa Institucional de Bolsas de Iniciação Científica (PIBIC/UFRJ-CNPq) a concessão de bolsas de IC.

\section{REFERÊNCIAS}

1. Serpe. A.; Artizzu, F.; Mercuri, M. L.; Pilia, L.; Deplano, P.; Coord. Chem. Rev. 2008, 252, 1200.

2. Frota Neto, J. Q.; Ruwaard, J. M. B.; van Nunen, J. A. E.; Heck, E. V.; Int. J. Prod. Econ. 2008, 111, 195.

3. Hagelüken, C.; Corti, C. W.; Gold Bull. 2010, 43, 209.

4. Wagner, T. P.; Waste Manage. 2009, 29, 3014.

5. Viktor, M.; Revista Galileu, n. 218, julho 2009, seção ambiente.

6. Computer and the Environment: Understanding and Managing Their Impacts, The United Nations University, Kluwer: Amsterdam, 2004.

7. Chade, J.; O Estado de São Paulo, São Paulo, seção notícias, edição de 23/02/2010.

8. Brasil; Lei Federal 12.305/10 (Política Nacional de Resíduos Sólidos), 02/08/2010, Diário Oficial da União, 03/08/2010.

9. Yellishetty, M.; Mudd, G. M.; Ranjith, P. G.; Tharumarajah, A.; Environ. Sci. Policy 2011, 14, 650.

10. Gerbase, A. E.; Oliveira, C. R.; Quim. Nova 2012, 35, 1486.

11. Ogunseitan, O. A.; Schoenung, J. M.; Sapheres, J. D. M.; Shapiro, A. A.; Science 2009, 326, 670.

12. Yamane, L. H.; Moraes, V. T.; Espinosa, D. C. R.; Tenório, J. A. S.; Waste Manage. 2011, 31, 2553.

13. Lee, C. H.; J. Hazard. Mater. 2004, 114, 93.

14. Duan, H.; Hou, K.; Li, J.; Zhu, X.; J. Environ. Manage. 2011, 92, 392.

15. Guo, J.; Xu, Z.; J. Hazard. Mater. 2009, 168, 567.

16. Huang, K.; Guo, J.; Xu, Z. M.; J. Hazard. Mater. 2009, 164, 399.

17. Zhou, Y. H.; Qiu, K. Q.; J. Hazard. Mater. 2010, 175, 823.

18. Lim, S.; Kang, D.; Ogunseitan, O, A.; Schoenung, J. M.; Environ. Sci. Technol. 2011, 45, 320.

19. Guo, C.; Wang, H.; Liang, W.; Fu, J.; Yi, K.; Waste Manage. 2011, 31, 2161.

20. Wang, F.; Huisman, J.; Meskers, C. E. M.; Schluep, M.; Stevels, A.; Hagelüken, C.; Waste Manage. 2012, 32, 2134.

21. Tunuck, A.; Stazi, V.; Akcil, A.; Yazici, E. Y.; Deveci, H.; Miner. Eng. 2012, 25, 28.

22. Kasper, A. C.; Berselli, G. B. T.; Freitas, B. D.; Tenório, J. A. S.; Bernardes, A. M.; Veit, H. M.; Waste Manage. 2011, 31, 2536.

23. Zheng, Y. H.; Shen, Z. G.; Cai, C. J.; Ma, S. L.; Xing, Y. S.; J. Hazard. Mater. 2009, 163, 600.

24. Guo, J.; Rao, Q. L.; Xu, Z. M.; J. Hazard. Mater. 2008, 153, 728.

25. Guo, J.; Guo, J. Y.; Cao, B.; Tang, Y. E.; Xu, Z. M.; J. Hazard. Mater. 2009, 163, 1019.

26. Li, J.; Lu, H.; Guo, J.; Xu, Z.; Zhou, Y.; Environ. Sci. Technol. 2007, 41, 1995. 
27. Xu, Z.; Li, J.; Zhou, Y.; J. Electrost. 2007, 65, 233.

28. Xu, Z.; Wu, J., Li, J.; Environ. Sci. Technol. 2008, 42, 5272.

29. Li, J.; Lu, H. Z.; Xu, Z. M.; Zhou, Y. H.; J. Hazard. Mater. 2008, 154, 331.

30. Wu, J.; Li, J.; Xu, Z. M.; J. Hazard. Mater. 2008, 159, 230.

31. De Marco, I.; Caballero, B. M.; Chomôn, M. J.; Laresgoiti, M. F.; Torres, A.; Fernández, G.; Arnaiz, S.; J. Anal. Appl. Pyrolysis 2008, 82, 179.

32. Moura, F. P.; Oliveira, R. S.; Afonso, J. C.; Vianna, C. A.; Mantovano, J. L.; Quim. Nova 2012, 35, 1271.

33. Marinho, R. S.; Afonso, J. C.; Cunha, J. W. S. D.; J. Hazard. Mater. 2010, 179, 488.

34. Chharlot, G.; Les Réactions Chimiques em Solutions Aquese, $7^{\mathrm{a}} \mathrm{ed}$., Masson: Paris, 1983, cap. VIII a XV.
35. Ohlweiler, O. A.; Química Analítica Quantitativa, $3^{\mathrm{a}}$ ed., Livros Técnicos e Científicos: Rio de Janeiro, 1981, vol. 1, p. 283-287.

36. Afonso, J. C.; Noronha, L. A.; Felipe, R. P.; Freidinger, N.; Quim. Nova 2003, 26, 602 .

37. Lima, R. M. G.; Wildhagen, G. R. S.; Cunha, J. W. S. D.; Afonso, J. C.; J. Hazard. Mater. 2009, 161, 1560.

38. Associação Brasileira de Normas Técnicas; NBR 10004, ABNT: Rio de Janeiro, 2004.

39. Brasil; Resolução do Conselho Nacional de Meio Ambiente (CONAMA) no 430, de 13/05/2011, Diário Oficial da União, 16/05/2011.

40. Brasil; Resolução do Conselho Nacional de Meio Ambiente (CONAMA) $n^{\circ}$ 264, de 26/08/1999, Diário Oficial da União, 20/03/2000.

41. Farias, L. A.; Fávaro, D. I. T.; Quim. Nova 2011, 34, 1089. 
PROCESSAMENTO DE PLACAS DE CIRCUITO IMPRESSO DE EQUIPAMENTOS ELETROELETRÔNICOS DE PEQUENO PORTE

Sérgio de Souza Henrique Júnior, Felipe Pereira de Moura, Roger de Souza Correa e Júlio Carlos Afonso*

Departamento de Química Analítica, Instituto de Química, Universidade Federal do Rio de Janeiro, Av. Athos da Silveira Ramos, 149, B1. A, 21941-909 Rio de Janeiro - RJ, Brasil

\section{Cláudio Augusto Vianna e José Luiz Mantovano}

Departamento de Química e Materiais Nucleares, Instituto de Engenharia Nuclear, Rua Hélio de Almeida, 75, 21941-906 Rio de Janeiro - RJ, Brasil

Placa de circuito impresso

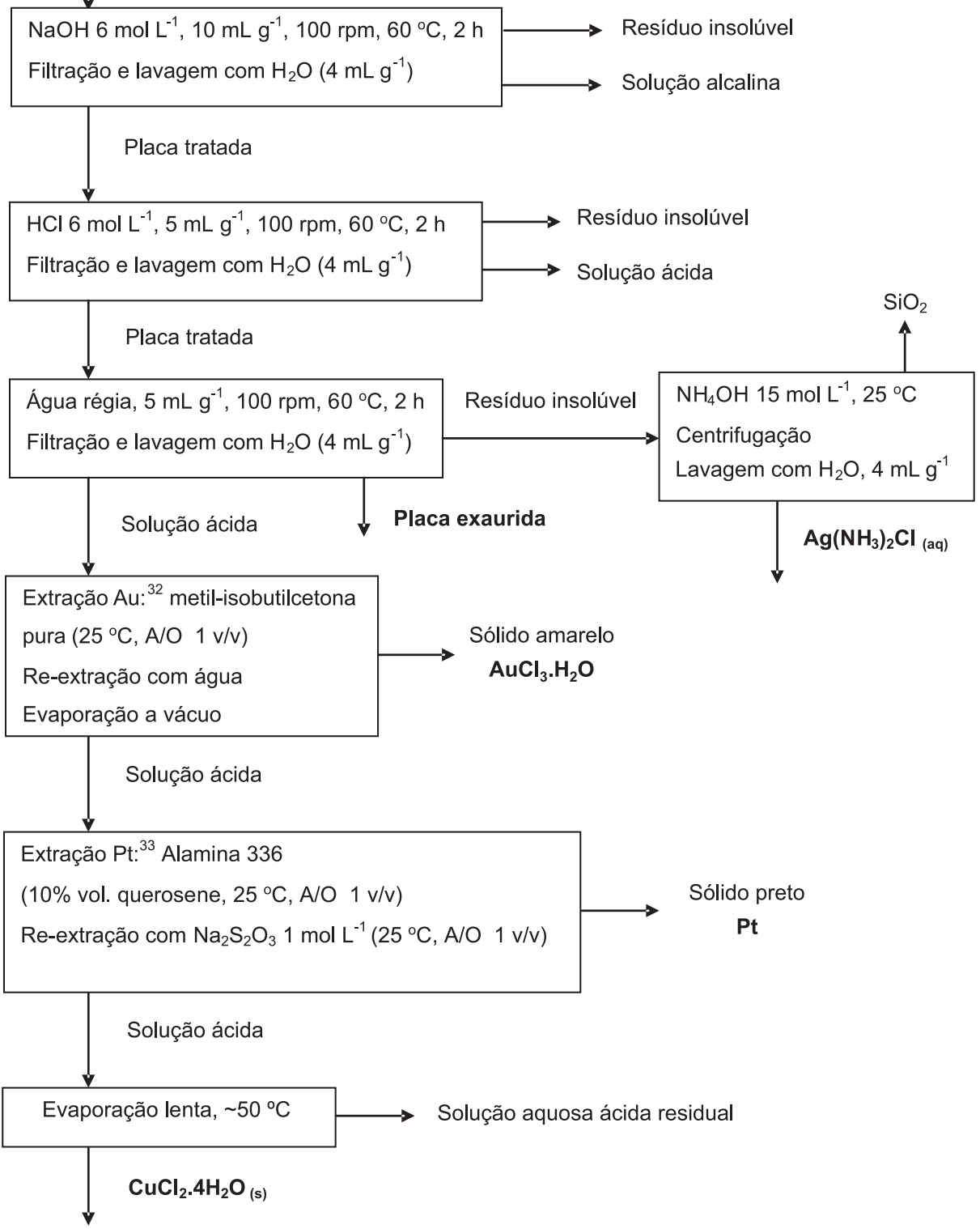

Figura 1S. Fluxograma completo de processamento de placas de circuito impresso (PCI) de telefones celulares 